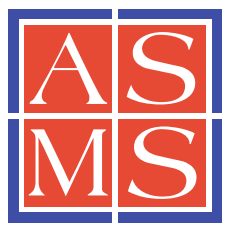

\title{
N-(1-Naphthyl) Ethylenediamine Dinitrate: A New Matrix for Negative Ion MALDI-TOF MS Analysis of Small Molecules
}

\author{
Rui Chen, ${ }^{1,2}$ Suming Chen, ${ }^{1,2}$ Caiqiao Xiong, ${ }^{1,2}$ Xunlei Ding, ${ }^{3}$ Chih-Che $\mathrm{Wu},{ }^{4}$ \\ Huan-Cheng Chang, ${ }^{5}$ Shaoxiang Xiong, ${ }^{1,2,6}$ Zongxiu Nie ${ }^{1,2,6}$
}

${ }^{1}$ Key Laboratory of Analytical Chemistry for Living Biosystems, Institute of Chemistry, The Chinese Academy of Sciences, Beijing, 100190, China

${ }^{2}$ Beijing National Laboratory for Molecular Sciences, Beijing, 100190, China

${ }^{3}$ State Key Laboratory for Structural Chemistry of Unstable and Stable Species, Institute of Chemistry,

The Chinese Academy of Sciences, Beijing, 100190, China

${ }^{4}$ Department of Applied Chemistry, National Chi Nan University, Puli, Nantou, 545, Taiwan

${ }^{5}$ Institute of Atomic and Molecular Sciences, Academia Sinica, Taipei, 106, Taiwan

${ }^{6}$ Beijing Center for Mass Spectrometry, Beijing, 100190, China

\begin{abstract}
An organic salt, $\mathrm{N}$-(1-naphthyl) ethylenediamine dinitrate (NEDN), with rationally designed properties of a strong UV absorbing chromophore, hydrogen binding and nitrate anion donors, has been employed as a matrix to analyze small molecules $(m / z<1000)$ such as oligosaccharides, peptides, metabolites and explosives using negative ion matrix-assisted laser desorption/ ionization time-of-flight mass spectrometry (MALDI-TOF MS). Compared with conventional matrixes such as $\alpha$-cyano-4-hydroxycinnamic acid (CCA) and 2,5-dihydroxybenzoic acid (DHB), NEDN provides a significant improvement in detection sensitivity and yields very few matrixassociated fragment and cluster ions interfering with MS analysis. For low-molecular-weight saccharides, the lowest detection limit achieved ranges from 500 amol to $5 \mathrm{pmol}$, depending on the molecular weight and the structure of the analytes. Additionally, the mass spectra in the lower mass range $(\mathrm{m} / \mathrm{z}<200)$ consist of only nitrate and nitric acid cluster ions, making the matrix particularly useful for structural identification of oligosaccharides by post-source decay (PSD) MALDI-MS. Such a characteristic is illustrated by using maltoheptaose as a model system. This work demonstrates that NEDN is a novel negative ion-mode matrix for MALDI-MS analysis of small molecules with nitrate anion attachment.
\end{abstract}

Key words: $N$-(1-naphthyl) ethylenediamine dinitrate, Negative ion-mode matrix, MALDI MS, Small molecule analysis

Electronic supplementary material The online version of this article (doi:10.1007/s13361-012-0421-z) contains supplementary material, which is available to authorized users.

Correspondence to: Chih-Che Wu; e-mail: wcche@ncnu.edu.tw, Zongxiu Nie; e-mail: znie@iccas.ac.cn

\section{Introduction}

$\mathrm{M}$ atrix-assisted laser desorption/ionization time-of-flight mass spectrometry (MALDI-TOF MS) has the characteristics of easy sample preparation, rapid analysis, high sensitivity, and, most notably, tolerance of contaminants in analysis. Since 1980s [1, 2], it has been widely used to analyze 
large molecular weight compounds such as proteins [3], polysaccharides [4], DNA/RNA [5], polymers [6], and even bacteria [7]. Although the high-throughput nature of MALDIMS makes it a powerful tool for the analysis of those molecules, its application in the field of small molecules has been rather limited because commonly used organic matrixes $[8,9]$ always produce a large number of matrix-associated interfering ions that obscure the detection of analytes in the low $\mathrm{m} / \mathrm{z}$ region.

A number of inorganic materials have been developed as alternative matrixes to avoid the drawbacks of using conventional organic matrixes [10-17]. Although low fragmentation of matrix ions can be attained with these techniques, several experimental factors, including surface morphology and laser fluence, need to be optimized simultaneously to achieve a reliable analysis. Recently, conjugated amino aromatic systems have been explored as matrixes for negative ion MALDI [18-24]. For example, 9aminoacridine was used as an efficient matrix to analyze low molecular compounds $[18,20,21]$. Owing to the presence of matrix-related ions in the mass spectra, it makes spectral interpretation obscure. Based on Brønsted-Lowry acid-base theory, Shroff et al. reported the use of proton sponge as a superbasic matrix for MALDI analysis of acidic analytes from biological samples [22, 23]. Hence, there is a continued need to develop new matrix interference-free methods for the analysis of small molecules by MALDI MS.

In MALDI MS analysis of small molecules, a suitable MALDI matrix should meet a number of requirements, including (1) strong absorption at the applied laser wavelength, (2) efficient ionization/desorption of the analytes, (3) little or no fragmentation of analyte, and (4) devoid of matrix interfering ions in the low $m / z$ region $[25,26]$. Here, we report a rationally designed matrix, $N$-(1-naphthyl) ethylenediamine dinitrate (NEDN), for small molecule analysis by negative ion MALDI MS. Interestingly, NEDN preferentially forms intact molecular radical cation in the gas phase, producing no or little interfering fragment ions in the low mass region. Additionally, the generations of nitrate ions during laser desorption/ionization (LDI) allow for detection of small molecules by anion attachment. These unique physical and chemical properties meet the above four requirements of being an effective matrix. The applicability of NEDN in performing small molecule analysis is demonstrated.

\section{Experimental}

$N$-(1-naphthyl) ethylenediamine dihydrochloride (NEDC) was purchased from Tianjin Xinbote Chemical Engineering (Tianjin, China). Silver nitrate, ethanol, methanol, and acetonitrile were obtained from Beijing Chemical Engineering (Beijing, China). Stachyose and pentafluorobenzoic acid were from Acros Organics (Morris Plains, NJ, USA). All chemicals were used in the highest purity and without further purification. The deionized water used was purified with a Mill-Q water system from Millipore (Milford, MA, USA).

\section{Preparation of Matrix and Sample Solutions}

To prepare NEDN, NEDC, and silver nitrate (at a molar ratio of 1:2) were dissolved together in ethanol/water ( $\mathrm{vol} / \mathrm{vol}$, 30:70). After $10 \mathrm{~min}$, silver chloride gradually precipitated, according to the reaction:

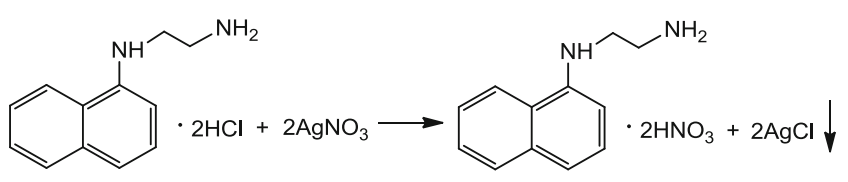

The $\mathrm{AgCl}_{(\mathrm{s})}$ precipitates were then removed by centrifugation at $5000 \mathrm{rpm}$ for $15 \mathrm{~min}$ and filtration with filter paper. The newly formed NEDN solution was purified once by passing through cation ion exchange resins (part no. 732; Jiangsu Linhai Resin Technology, China) and the effluent was used as the matrix solution without further treatment.

Matrix solutions were prepared at a concentration of $100 \mathrm{mM}$ in ethanol/water (vol/vol, 30:70) and were diluted to desired concentrations as required. Except hexahydro1,3,5-trinitro-1,3,5-triazine (RDX), which was prepared in tetrahydrofuran, and pentafluorobenzoic acid, uric acid, ascorbic acid, which were prepared in pure ethanol, the other analytes were all dissolved in methanol/water ( vol/vol, 50:50) to a final concentration of $10 \mathrm{mM}$ and diluted to desired concentrations prior to MS analysis.

\section{Optical Characterization}

Ultraviolet-visible (UV-Vis) absorption spectra of NEDN and DHB were analyzed using a TU-1900 double beam UV-Vis spectrophotometer (Purkinje General Instrument, Beijing, China) in a $1-\mathrm{cm}$ path length cuvette at room temperature. Micrographs of NEDN crystallites were acquired with an S-4800 scanning electron microscope (Hitachi, Tokyo, Japan).

\section{Mass Spectrometry}

All MS measurements were performed with either a Microflex or a Biflex III MALDI-TOF mass spectrometer (Bruker Daltonics, Bremen, Germany) equipped with a nitrogen laser $(\lambda=337 \mathrm{~nm})$. Mass spectra (20 summed shots) were acquired in the reflector mode with a $19 \mathrm{kV}$ accelerating voltage and a $20 \mathrm{kV}$ reflector voltage. The laser pulse energy was set marginally above the threshold of ionization to avoid fragmentation. Prior to MS analysis, matrix solution and analyte solution were mixed at an equal volume, and $1 \mu \mathrm{L}$ of the mixed solution was spotted on the MALDI target plate. After being dried in air, the samples were subjected to analysis.

\section{Results and Discussion}

\section{NEDN as a Negative Ion-Mode Matrix for Small Molecule Analysis}

In analysis of small molecules by MALDI MS, the matrix plays a key role since it serves not only as a laser light 
energy absorbent and a thermal energy transporter, but also as a potential charge donor. For the typical MALDI matrixes, such as DHB, there is a significantly large absorption in the UV region because of its unique benzoic acid core structure [9]. $N$-(1-naphthyl) ethylenediamine dinitrate contains a naphthalene core, which acts as a strong UV absorbing chromophore and shows a strong and broad absorption band centered at $324 \mathrm{~nm}$. The molar extinction coefficient $\left(\varepsilon=7.10 \times 10^{3} \mathrm{M}^{-1} \mathrm{~cm}^{-1}\right)$ of NEDN is about 3-fold larger than that of DHB $\left(\varepsilon=2.17 \times 10^{3} \mathrm{M}^{-1} \mathrm{~cm}^{-1}\right)$ at the applied laser wavelength of $337 \mathrm{~nm}$ (Figure 1S in Supplementary Material). This important feature makes NEDN a good MALDI matrix in the first place.

It is of interest to note that the positive ion LDI spectrum of the NEDN shows no signals associated with protonated $\mathrm{N}$-(1-naphthyl) ethylenediamine (NED) at $\mathrm{m} / z$ 187.12 [22, 23] (Figure $2 \mathrm{~S}$ in Supplementary Material). Instead, only one intense peak corresponding to the NED radical cation appears at $\mathrm{m} / \mathrm{z} 186.12$ [27]. This unique property allows effective desorption upon laser irradiation to proceed but with little background interference due to matrix fragmentation. The negative ion mass spectrum of NEDN is remarkably simple and essentially free of background signals except two characteristic peaks at $\mathrm{m} / \mathrm{z} 61.98$ and 124.98, corresponding to $\left[\mathrm{NO}_{3}\right]^{-}$and $\left[\mathrm{HNO}_{3}+\mathrm{NO}_{3}\right]^{-}$, respectively. Interestingly, neither molecular ions corresponding to NED nor their fragments could be detected in its mass spectrum. It can be envisioned that the NED group in the matrix absorbs the laser photon energy and transfers the energy to the analytes, and the nitrate acid serves as an anion donor in the MALDI process.

Good cocrystallization of matrix and analyte is one of the most important factors in producing effective laser desorption/ionization [28]. In this process, solvent plays a crucial role and profoundly modulates the analyte-matrix interactions, resulting in the increase or decrease of the MALDI ion signals [29-31]. For example, when using NEDN as a matrix, the mass spectrum of sucrose shows only a very weak signal if the matrix is dissolved in solvent such as pure water, methanol, or ethanol. Titration experiments at different ratios of ethanol/water led to an optimal condition of ethanol:water $=30: 70(\mathrm{vol} / \mathrm{vol})$ (Figure 3S in Supplementary Material).

In addition to the matrix solvent, we also found that the composition of the analyte solvent strongly influences the peak intensity of the nitrate adduct ions in MALDI mass spectra. Using sucrose as the test compound, when the sample was dissolved in 50:50 methanol/water, the highest signal intensity can be gained (Figure 4S in Supplementary Material). We therefore selected 30:70 ethanol/water as the matrix solvent and 50:50 methanol/water as the analyte solvent in the subsequent experiments.

We studied the effect of the molar ratio of matrix to analyte on the signal intensity of the adduct ions [32]. In conventional MALDI-TOF MS analysis, the typical matrix-toanalyte ratio varies from 1500:1 to $3000: 1$, depending on the species and molecular weights of analytes [33]. Surprisingly, we found that the optimal molar ratio used for this benchmark system was 10:1 (Figure 5S in Supplementary Material). This is very different from the widely accepted idea that a very high matrix/analyte ratio is required for effective laser desorption/ ionization. The observation of a relatively low matrix/analyte ratio suggests that the NEDN matrix behaves very differently from the conventional DHB matrix. The nitrate anion donating capability of the NEDN to the analyte is very effective, which minimizes the required amount of matrix for charge transfer ionization. When the ratio is greater than 100:1, the signals of $\left[\mathrm{NO}_{3}\right]^{-}$and $\left[\mathrm{HNO}_{3}+\mathrm{NO}_{3}\right]^{-}$ions are too strong, making it very difficult to detect the nitrate adduct ions in the mass spectra, presumably due to the ion competition effect. However, when the ratio is less than 1:5, there is not enough matrix to assist laser desorption/ionization of analyte and the cocrystallization of matrix with analyte is poor. It is considered that because of the free NH hydrogen bonds derived from primary and secondary amines, NEDN can provide additional hydrophilic bonds for analyte to be embedded and isolated. Hence, the NEDN can easily form molecular complexes with analytes in the matrix, which facilitates the process of laser desorption/ ionization of analyte.

Figure 1 shows the MALDI mass spectra of $5 \mathrm{nmol}$ of oligosaccharides and $5 \mathrm{nmol}$ of di- and tetra-peptides using NEDN as the matrix, respectively. The peaks at $\mathrm{m} / \mathrm{z} 242.05$, 404.10 , and 728.21 are assigned to the nitrate adducts of D-galactose, sucrose, and stachyose, respectively. In comparison to the mass spectrum of pure NEDN, only the nitrate-attached molecular anion was newly found in the spectra. There is no fragmentation of the analyte and the
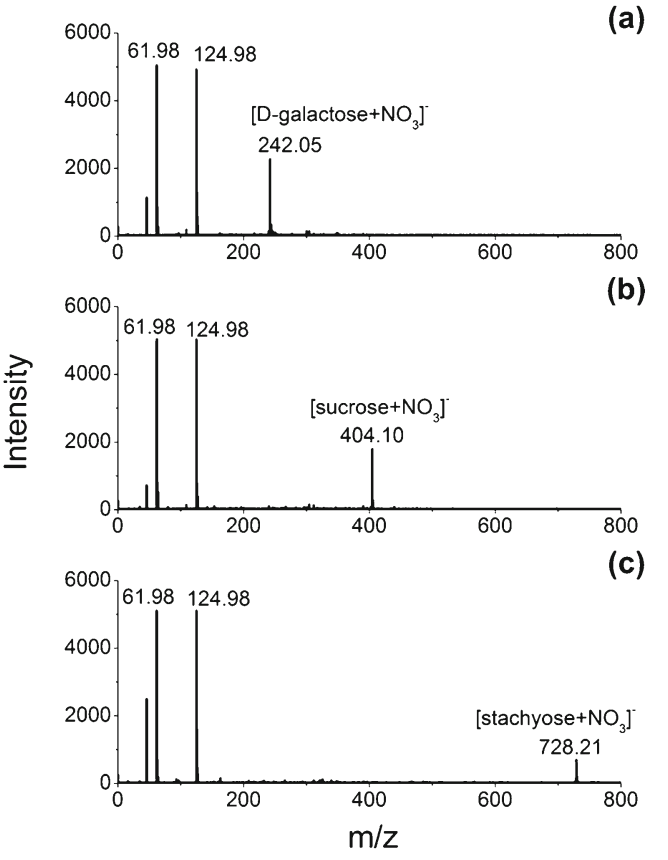

Figure 1. MALDI-TOF mass spectra of (a) D-galactose, (b) sucrose, (c) stachyose obtained with NEDN as matrix. The spectra were acquired in the negative ion mode 
NEDN matrix, making the low-mass range $(\mathrm{m} / \mathrm{z} 150-1000)$ a clear window for observation of small oligosaccharides. From a series of experiments with NEDN as matrix, we conclude that small neutral saccharides such as sucrose can form anionic adducts with nitrates. These results also show that there is little or no fragmentation of the analytes and matrix molecules, illustrating the excellent performance of NEDN in MALDI-MS analysis especially in the low-mass region.

The matrixes, CCA and DHB, have long been regarded as the preferred matrixes for MALDI-MS analysis of carbohydrates $[8,9,31,34]$. To illustrate the advantage of using NEDN over CCA and DHB for MALDI-MS analysis of small molecules, we conducted a comparative study using sucrose as test compounds (Figure 6S in Supplementary Material). The signals of sucrose were not detected in the negative ion mass spectra when CCA or DHB were used as matrixes. With the use of NEDN as a matrix, the analyte is readily detected as the nitrate adducts with high peak intensities, sensitivity, and, more importantly, the interference from matrix ions is completely eliminated. No fragment ions of the analytes are observed, suggesting that NEDN is an effective negative ion-mode matrix for MALDI-TOF MS analysis of small molecules.

Very recently, we have reported that NEDC can be used as an effective matrix to analyze small molecules with chloride-attached product [27]. It is instructive to compare the efficacy of adduct ion formation between $\mathrm{Cl}^{-}$and $\mathrm{NO}_{3}{ }^{-}$ with the small molecules investigated herein. In this experiment, an equal molar ratio of NEDN and NEDC was mixed and used as a binary anion donor matrix for the analysis of sucrose and Gly-Leu-Tyr. The mass spectrum of pure binary matrix (NEDN/NEDC; 1:1 molar ratio) shows strong signals of $\left[\mathrm{NO}_{3}\right]^{-}$and $\left[\mathrm{HNO}_{3}+\mathrm{NO}_{3}\right]^{-}$, but neither $[\mathrm{Cl}]^{-}$nor $[\mathrm{HCl}+\mathrm{Cl}]^{-}$appears in the spectrum (Figure $7 \mathrm{~S}$ in Supplementary Material). Similar results were found in the mass spectra of both analytes, indicating that the nitrate adducts of both oligosaccharides and peptides can be generated more readily than their corresponding anionic chloride adducts. The finding could be explained by the fact that the gas-phase basicity of chloride ions is higher than that of nitrate ions, and this places a tighter restriction on the ability to observe nitrate adducts in MALDI MS $[19,35,36]$.

The exceptionally few interfering low-mass ions in the mass window below $\mathrm{m} / \mathrm{z} 150$ can enable NEDN as an effective matrix for MALDI-MS analysis of small molecules. Hexahydro-1,3,5-trinitro-1,3,5-triazine is a reduced symmetrical triazine ring compound, which is explosively unstable because of the presence of three N-nitro substituents. The spectrum shows that RDX can form a nitrate anionic adduct at $\mathrm{m} / \mathrm{z} 284.02$ in the process of laser desorption/ionization of NEDN (Figure 2). When NEDN was used as the matrix to analyze pentafluorobenzoic acid, nitrate adducts, deprotonated and decarboxyl ions can all be
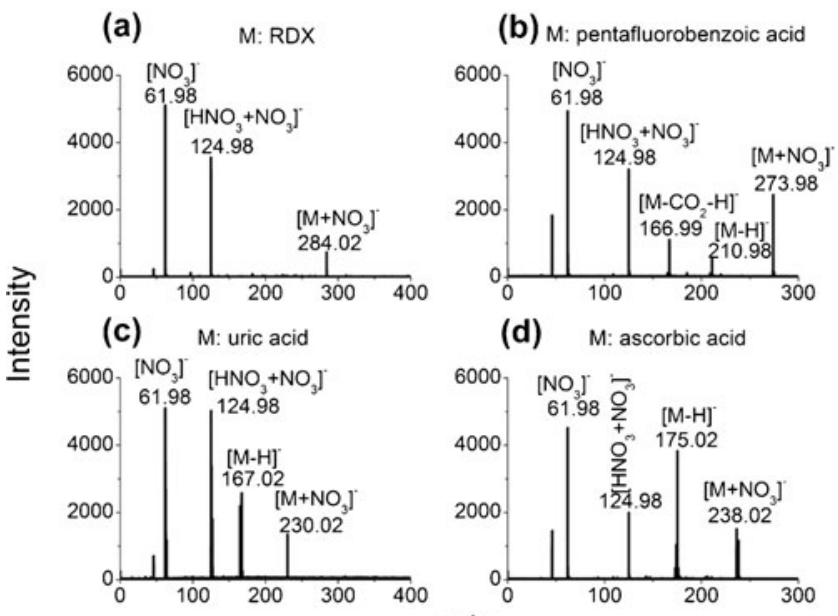

(d) M: ascorbic acid

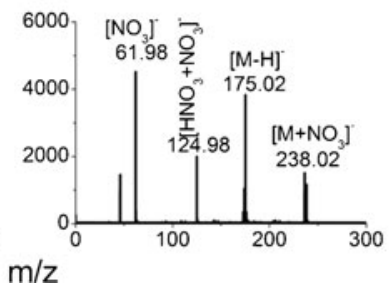

Figure 2. MALDI-TOF mass spectra of (a) RDX, (b) pentafluorobenzoic acid, (c) uric acid, and (d) ascorbic acid obtained with NEDN as matrix. RDX was prepared in tetrahydrofuran at a concentration of $10 \mathrm{mM}$, the other three acids in pure ethanol at a concentration of $10 \mathrm{mM}$, and the matrix in 30:70 ethanol/water. The spectra were acquired in the negative ion mode

found at $\mathrm{m} / \mathrm{z} 273.98,210.98$ and 166.99 in its mass spectrum. The reason that the signal of the decarboxylated ion is stronger than that of the deprotonated ion is because the bond length $(1.54 \AA)$ between the benzene ring and the carbon atom on the carboxyl group is longer than that of $-\mathrm{C}-\mathrm{O}$ $(1.41 \AA)$ in the carboxyl group and, hence, the $-\mathrm{COOH}$ loss can readily occur. When NEDN was used as a matrix to analyze metabolites such as uric acid and ascorbic acid, both deprotonated ions and nitrate adducts could be observed in their mass spectra. They reside at $\mathrm{m} / \mathrm{z} 167.02$ and 230.02 for uric acid, and at $\mathrm{m} / \mathrm{z} 175.02$ and 238.02 for ascorbic acid, respectively.

We further extensively studied the feasibility of generating anionic nitrate adducts with NEDN as the new matrix to facilitate the analysis of small molecules in the negative ion mode of MALDI-MS using a variety of low molecular weight compounds including oligosaccharides, peptides, explosives, and metabolites. The laser power was adjusted to a value slightly above the desorption/ionization threshold, and each mass spectrum was obtained by averaging 20 laser shots scanned across the sample spot. The samples were extensively diluted with 50:50 methanol/water solution to desired concentrations prior to analysis. Taking the analysis of glucose as an example, we defined a positive detection of nitrate-attached glucose ions based on the peak detection criteria of above a signal-to-noise (S/N) to 3 (Figure 3) [29, 37-39]. Experimentally determined limit of detections (LODs) for the above mentioned analytes were listed in the Supplementary Table 1 . The detection limit of glucose was 500 amol, significantly lower than that obtained using conventional DHB matrix [40]. The NEDN-assisted LDI process is shown to achieve highly sensitive detection for 


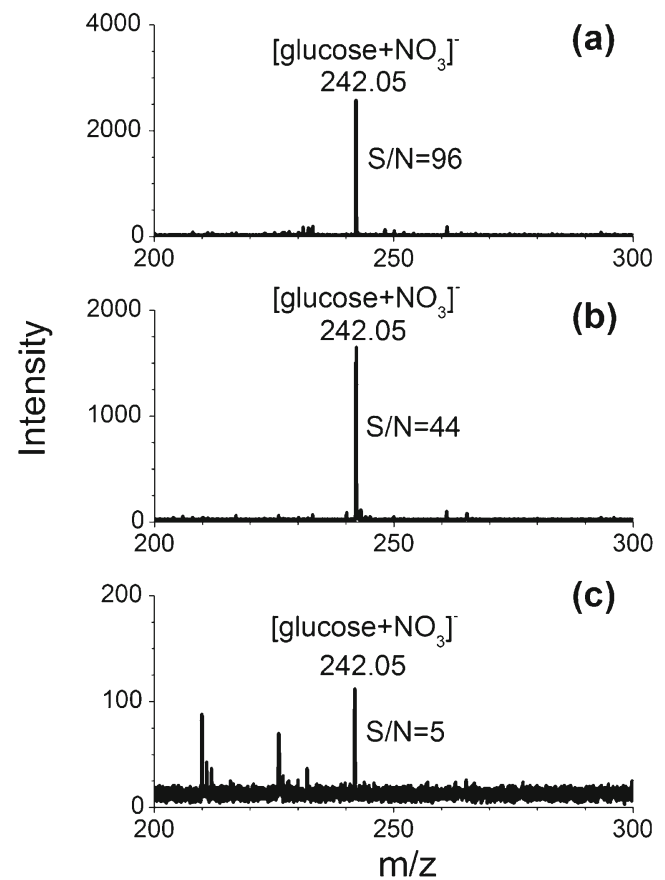

Figure 3. MALDI-TOF mass spectra of glucose obtained with NEDN as matrix. The amounts of glucose deposited on the MALDI plate are (a) $500 \mathrm{pmol}$, (b) $500 \mathrm{fmol}$, and (c) 500 amol, respectively. Glucose was only detected as nitrate attached anionic adduct numerous small molecules with molecule weight below $1000 \mathrm{Da}$, and is expected to find potential applications in the biological and biomedical research.

\section{Structural Characterization of Oligosaccharide}

Sensitive detection of small oligosaccharides with NEDN matrix can be achieved in the negative-ion mode MALDI MS analysis. In this mode, the background interference derived from sample and matrix fragmentation is minimized, which is well-suited for post-source decay (PSD) fragment ion mass analysis by MALDI mass spectrometry. $\mathrm{N}$-(1naphthyl) ethylenediamine dinitrate was thus used as a negative-ion mode matrix in the structural characterization of maltoheptaose using PSD-MALDI mass spectrometry. The signals of analyte at $\mathrm{m} / \mathrm{z} 1214.4$ were attributed to the nitrate adduct of oligosaccharide in the negative mode MALDI mass spectrum. To further characterize the structure of the maltoheptaose, the adduct ions $\left[\mathrm{M}+\mathrm{NO}_{3}\right]^{-}$were selected as precursors for PSD fragmentation. The negative ion mode PSD spectrum of maltoheptaose with NEDN matrix was shown in Figure 4. Domon and Costello nomenclature [41] is used to describe the MALDI-TOF/ TOF fragmentation patterns of maltoheptaose. Subscripts refer to the number of rings retained, and superscripts indicate cleavages within carbohydrate rings. A nonreducing
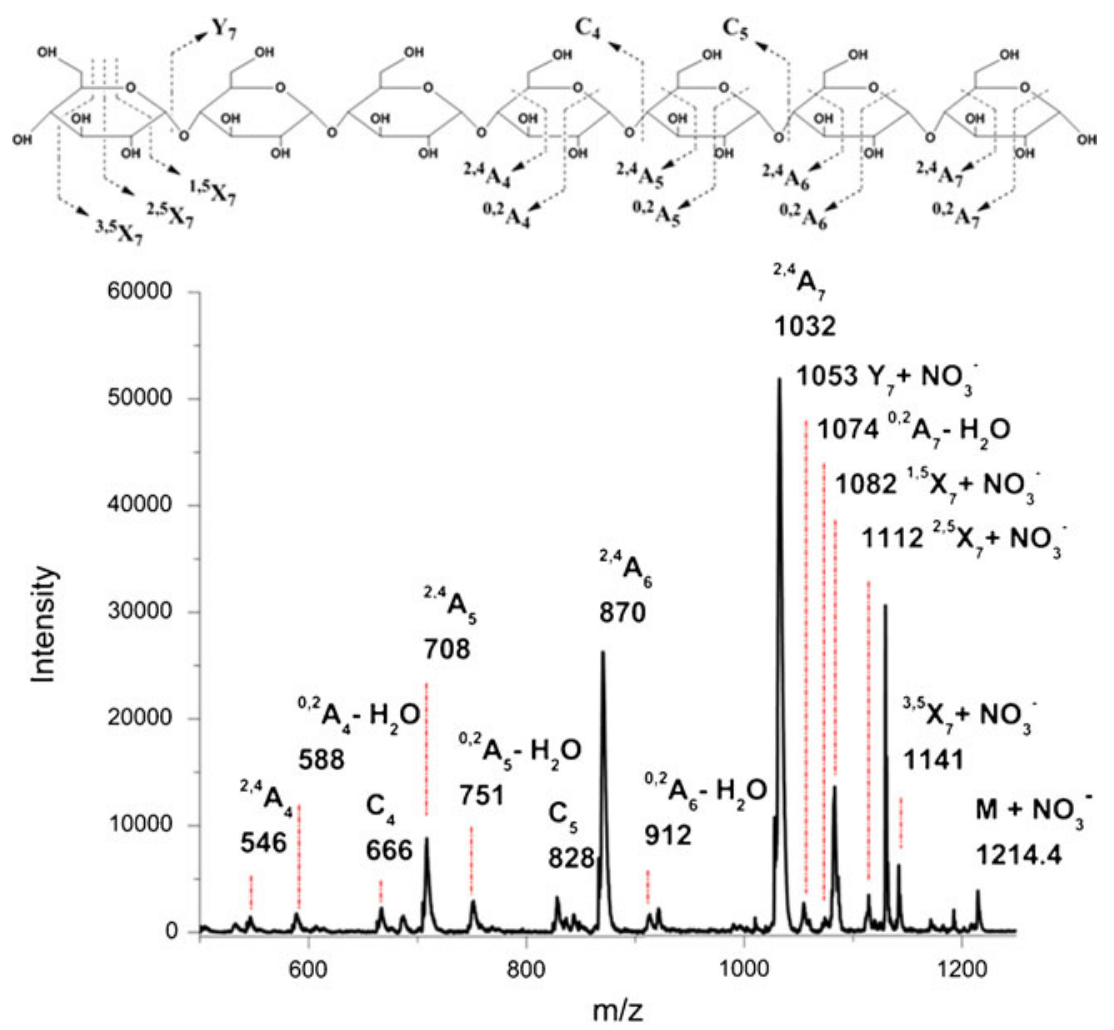

Figure 4. Negative ion mode MALDI-PSD mass spectrum of maltoheptaose with NEDN as matrix. Nitrate attached maltoheptaose ions are selected as MS/MS precursors 
end without the glycosidic oxygen is referred to as an $A_{n}$ ion and a nonreducing ion that retains the glycosidic oxygen is referred to as a $C_{n}$ ion. If the anions are retained by the reducing end of a glycosidic cleavage ion, $Y_{n}$ and $Z_{n}$ ions are used to designate the ions. The use of two superscripts $k$ and $l$ (i.e., ${ }^{k, l} A_{i}$ and ${ }^{k, l} X_{j}$ ) to indicate the sugar ring bonds that have been broken. For $A_{i}$, the situation is more complex, and the loss of water needs to be specified (e.g., $\left[{ }^{0,2} A_{4}-H_{2} O\right]$, $\left[{ }^{0,2} A_{5}-H_{2} O\right],\left[{ }^{0,2} A_{6}-H_{2} O\right]$ and $\left[{ }^{0,2} A_{7}-H_{2} O\right]$ observed at $m / z 588,751,912$ and 1074, respectively), while for $X_{i}$ and $Y_{i}$, nitrate adducts are readily produced (e.g., $\left[\mathrm{Y}_{7}+\mathrm{NO}_{3}\right]^{-}, \quad\left[{ }^{1,5} \mathrm{X}_{7}+\mathrm{NO}_{3}\right]^{-}, \quad\left[{ }^{2,5} \mathrm{X}_{7}+\mathrm{NO}_{3}\right]^{-}$, and $\left[3,5 X_{7}+N O_{3}\right]^{-}$at $\mathrm{m} / \mathrm{z} 1053,1082,1112$, and 1141 , respectively). The PSD fragmentation spectrum of nitrate attached maltoheptaose ions revealed abundant of crossring fragmentation. This observation may be explained by the nitrate attachment of maltoheptaose via hydrogen bond formation increased the activation energy required for glycosidic bond cleavages and resulted in the increase of cross-ring fragmentation ions. The obtained mass spectrum was essentially matrix interference-free, which greatly facilitated the fragmentation pattern assignment in the low mass region. The NEDN is demonstrated to be a suitable matrix for sensitive analysis of structurally informative fragment ions derived by negative ion PSD spectra of nitrate-attached adduct of oligosaccharides.

\section{Conclusion}

With the combined properties of strong UV absorption, hydrogen bonding, and nitrate anion donation capabilities, NEDN has been demonstrated to be useful as a negative ionmode matrix to analyze small molecules by MALDI MS. Compared with commonly used matrixes, such as CCA and DHB, NEDN is advantageous in that it allows straightforward mass spectrometric identification of analytes without resorting to high $\mathrm{m} / \mathrm{z}$ resolution and accuracy. We envision further applications of this new matrix, particularly in imaging of small molecules in biological tissues with MALDI-TOF MS [42].

\section{Acknowledgement}

The authors acknowledge support for this work by grants from the Chinese Academy of Sciences (grant YZ201126) and the National Natural Sciences Foundation of China (grants 21127901, 20927006, and 21175139). The authors also acknowledge support from the National Science Council and Academia Sinica of Taiwan. The authors thank Chia-Hong Lin for technical assistance.

\section{References}

1. Karas, M., Bachmann, D., Bahr, U., Hillenkamp, F.: Matrix-assisted ultraviolet laser desorption of nonvolatile compounds. Int. J. Mass Spectrom. Ion Processes 78, 53-68 (1987)
2. Tanaka, K., Akita, S., Yoshida, Y., Yoshida, T., Matsuo, T.: Protein and polymer analyses up to $\mathrm{m} / \mathrm{z} 100,000$ by laser ionization time-of-flight mass spectrometry. Rapid Commun. Mass Spectrom. 2, 151-153 (1988)

3. Chaurand, P., Luetzenkirchen, F., Spengler, B.: Peptide and protein identification by matrix-assisted laser desorption ionization (MALDI) and MALDI-post-source decay time-of-flight mass spectrometry. J. Am. Soc. Mass Spectrom. 10, 91-103 (1999)

4. Garrozzo, D., Impallomeni, G., Spina, E., Sturiale, L., Zanetti, F.: Matrix-assisted laser desorption/ionization mass spectrometry for polysaccharides. Rapid Commun. Mass Spectrom. 9, 937-941 (1995)

5. Guillonneau, F., Guieysse, A.L., Le Caer, J.P., Rossier, J., Praseuth, D.: Selection and identification of proteins bound to DNA triple-helical structures by combination of 2D-electrophoresis and MALDI-TOF mass spectrometry. Nucleic Acid Res. 29, 2427-2436 (2001)

6. Bahr, U., Deppe, A., Karas, M., Hillenkamp, F., Giessmann, U.: Mass spectrometry of synthetic polymers by UV-matrix-assisted laser desorption/ionization. Anal. Chem. 64, 2866-2869 (1992)

7. Bundy, J., Fenselau, C.: Lectin-based affinity capture for MALDI-MS analysis of bacteria. Anal. Chem. 71, 1460-1463 (1999)

8. Beavis, R.C., Chaudhary, T., Chait, B.T.: $\alpha$-Cyano-4-hydroxycinnamic acid as a matrix for matrix-assisted laser desorption mass spectrometry. Org. Mass Spectrom. 27, 156-158 (1992)

9. Strupat, K., Karas, M., Hillenkamp, F.: 2,5-Dihydroxybenzoic acid: a new matrix for laser desorption-ionization mass spectrometry. Int. J. Mass Spectrom. Ion Processes. 111, 89-102 (1991)

10. Wei, J., Buriak, J.M., Siuzdak, G.: Desorption/ionization mass spectrometry on porous silicon. Nature 399, 243-246 (1999)

11. Zhang, Q., Zou, H., Guo, Z., Zhang, Q., Chen, X., Ni, J.: Matrixassisted laser desorption/ionization mass spectrometry using porous silicon and silica gel as matrix. Rapid Commun. Mass Spectrom. 15, 217-223 (2001)

12. Go, E.P., Apon, J.V., Luo, G., Saghatelian, A., Daniels, R.H., Sahi, V., Dubrow, R., Cravatt, B.F., Vertes, A., Siuzdak, G.: Desorption/ ionization on silicon nanowires. Anal. Chem. 77, 1641-1646 (2005)

13. Wen, X., Dagan, S., Wysocki, V.H.: Small molecule analysis with silicon-nanoparticle-assisted laser desorption/ionization mass spectrometry. Anal. Chem. 79, 434-444 (2007)

14. Yan, H., Xu, N., Huang, W.Y., Han, H.M., Xiao, S.J.: Electroless plating of silver nanoparticles on porous silicon for laser desorption/ ionization mass spectrometry. Int. J. Mass Spectrom. 281, 1-7 (2009)

15. Gholipour, Y., Giudicessi, S.L., Nonami, H., Erra-Balsells, R.: Diamond, titanium dioxide, titanium silicon oxide, and barium strontium titanium oxide nanoparticles as matrixes for direct matrixassisted laser desorption/ionization mass spectrometry analysis of carbohydrates in plant tissues. Anal. Chem. 82, 5518-5526 (2010)

16. Pan, C., Xu, S., Hu, L., Su, X., Ou, J., Zou, H., Guo, Z., Zhang, Y., Guo, B.: Using oxidized carbon nanotubes as matrix for analysis of small molecules by MALDI-TOF MS. J. Am. Soc. Mass Spectrom. 16, 883-892 (2005)

17. Dong, X., Cheng, J., Li, J., Wang, Y.: Graphene as a novel matrix for the analysis of small molecules by MALDI-TOF MS. Anal. Chem. 82, 6208-6214 (2010)

18. Vermillion-Salsbury, R.L., Hercules, D.M.: 9-Aminoacridine as a matrix for negative mode matrix-assisted laser desorption/ionization. Rapid Commun. Mass Spectrom. 16, 1575-1581 (2002)

19. Cai, Y., Jiang, Y., Cole, R.B.: Anionic adducts of oligosaccharides by matrix-assisted laser desorption/ionization time-of-flight mass spectrometry. Anal. Chem. 75, 1638-1644 (2003)

20. Vaidyanathan, S., Goodacre, R.: Quantitative detection of metabolites using matrix-assisted laser desorption/ionization mass spectrometry with 9-aminoacridine as matrix. Rapid Commun. Mass Spectrom. 21, 2072-2078 (2007)

21. Becher, J., Muck, A., Mithöfer, A., Svatoš, A., Boland, W.: Negative ion mode matrix-assisted laser desorption/ionization time-of-flight mass spectrometric analysis of oligosaccharides using halide adducts and 9-aminoacridine matrix. Rapid Commun. Mass Spectrom. 22, 1153-1158 (2008)

22. Shroff, R., Svatoš, A.: Proton sponge: A novel and versatile MALDI matrix for the analysis of metabolites using mass spectrometry. Anal. Chem. 81, 7954-7959 (2009)

23. Shroff, R., Rulíšek, L., Doubský, J., Svatoš, A.: Acid-base-driven matrix-assisted mass spectrometry for targeted metabolomics. Proc. Natl. Acad. Sci. U.S.A. 106, 10092-10096 (2009)

24. Rohmer, M., Meyer, B., Mank, M., Stahl, B., Bahr, U., Karas, M.: 3 Aminoquinoline acting as matrix and derivatizing agent for MALDI MS analysis of oligosaccharides. Anal. Chem. 82, 3719-3726 (2010) 
25. Hillenkamp, F., Peter-Katalinic, J.: MALDI MS: A Practical Guide to Instrumentation, Methods and Applications. Wiley-VCH GmbH \& Co. KGaA, Weinheim (2007)

26. Hoffmann, E., Stroobant, V.: Mass Spectrometry Principles and Application, 3rd edn, pp. 33-39. John Wiley \& Sons, Chichester (2007)

27. Chen, R., Xu, W., Xiong, C., Zhou, X., Xiong, S., Nie, Z., Mao, L., Chen, Y., Chang, H.-C.: High-salt-tolerance matrix for facile detection of glucose in rat brain microdialysates by MALDI-mass spectrometry. Anal. Chem. 84, 645-649 (2012)

28. Mirza, S.P., Raju, P., Madhavendra, S.S., Vairamani, M.: 5-Amino-2mercapto-1,3,4-thiadiazole: a new matrix for the efficient matrixassisted laser desorption/ionization of neutral carbohydrates. Rapid Commun. Mass Spectrom. 18, 1666-1674 (2004)

29. Cohen, S.L., Chait, B.T.: Influence of matrix solution conditions on the MALDI-MS analysis of peptides and proteins. Anal. Chem. 68, 31-37 (1996)

30. Sun, G., Yang, K., Zhao, Z., Guan, S., Han, X., Gross, R.W.: Matrixassisted laser desorption/ionization time-of-flight mass spectrometric analysis of cellular glycerophospholipids enabled by multiplexed solvent dependent analyte-matrix interactions. Anal. Chem. 80, 75767585 (2008)

31. Tzeng, Y.-K., Zhu, Z., Chang, H.-C.: Alkali-hydroxide-doped matrices for structural characterization of neutral underivatized oligosaccharides by MALDI time-of-flight mass spectrometry. J. Mass Spectrom. 44, 375-383 (2009)

32. Zhang, S., Liu, J., Chen, Y., Xiong, S., Wang, G., Chen, J., Yang, G.: A novel strategy for MALDI-TOF MS analysis of small molecules. $J$. Am. Soc. Mass Spectrom. 21, 154-160 (2010)

33. Goldschmidt, R.J., Wetzel, S.J., Blair, W.R., Guttman, C.M.: Postsource decay in the analysis of polystyrene by matrix-assisted laser desorption/ionization time-of-flight mass spectrometry. J. Am. Soc. Mass Spectrom. 11, 1095-1106 (2000)
34. Harvey, D.J.: Matrix-assisted laser desorption/ionization mass spectrometry of carbohydrates. Mass Spectrom. Rev. 18, 349-451 (1999)

35. Gluckmann, M., Pfenninger, A., Kruger, R., Thierolf, M., Karas, M., Horneffer, V., Hillenkamp, F., Strupat, K.: Mechanism in MALDI analysis: surface interaction or incorporation of analytes? Int. J. Mass Spectrom. 210/211, 121-132 (2001)

36. Guan, B., Cole, R.B.: MALDI linear-field reflectron TOF post-source decay analysis of underivatized oligosaccharides: determination of glycosidic linkages and anomeric configurations using anion attachment. J. Am. Soc. Mass Spectrom. 19, 1119-1131 (2008)

37. Langley, G.J., Julie, M., Herniman, J.M., Townell, M.S.: 2B or not 2B, that is the question: further investigations into the use of pencil as a matrix for matrix-assisted laser desorption/ionization. Rapid Commun. Mass Spectrom. 21, 180-190 (2007)

38. Rohmer, M., Meyer, B., Mank, M., Stahl, B., Bahr, U., Karas, M.: 3Aminoquinoline acting as matrix and derivatizing agent for MALDI MS analysis of oligosaccharides. Anal. Chem. 82, 3719-3726 (2010)

39. Chen, W.T., Chiang, C.K., Lin, Y.W., Chang, H.T.: Quantification of captopril in urine through surface-assisted laser desorption/ionization mass spectrometry using 4-mercaptobenzoic acid-capped gold nanoparticles. J. Am. Soc. Mass Spectrom. 21, 864-867 (2010)

40. Yang, H.-J., Lee, A., Lee, M.-K., Kim, W., Kim, J.: Detection of small neutral carbohydrates using various supporting materials in laser desorption/ionization mass spectrometry. Bull. Korean Chem. Soc. 31, 35-40 (2010)

41. Domon, B., Costello, C.E.: A systematic nomenclature for carbohydrate fragmentations in FAB-MS/MS spectra of glycoconjugates. Glycoconj. J. 5, 397-409 (1988)

42. Jun, J.H., Song, Z., Liu, Z., Nikolau, B.J., Yeung, E.S., Lee, Y.J.: Highspatial and high-mass resolution imaging of surface metabolites of Arabidopsis thaliana by laser desorption-ionization mass spectrometry using colloidal silver. Anal. Chem. 82, 3255-3265 (2010) 